\title{
Candidíase oral como marcador de prognóstico em pacientes portadores do HIV
}

\author{
Valdinês Gonçalves dos Santos Cavassani ${ }^{1}$, \\ Jozias de Andrade Sobrinho², Maria da Graça \\ Naclério Homem³, Abrão Rapoport ${ }^{4}$
}

\section{Oral candidiasis as prognostic marker of HIV- infected patients}

\section{Resumo / Summary}

ntrodução: A candidíase oral é uma das doenças oportunistas mais fortemente associadas à infecção pelo Vírus da Imunodeficiência Humana (HIV). Vários relatos epidemiológicos enfatizam a prevalência da candidíase em pacientes HIV positivos e ressaltam a sua importância como marcador da progressão da doença e preditivo para o aumento da imunodepressão. Objetivo: Verificar as alterações estomatológicas em pacientes portadores do HIV tratados no Hospital Heliópolis - São Paulo, Brasil e comparar com a literatura. Forma de Estudo: Retrospectivo clínico nãorandomizado. Casuística e Método: Foram analisados 431 pacientes HIV+/AIDS (298 homens e 133 mulheres) no Hospital Heliópolis - São Paulo, Brasil, no período de 1995 a 2001. Resultados: A idade média mais comum foi dos 31 aos 40 anos (47,10\%); a via de contágio mais comum foi a sexual $(71,26 \%)$. Dentre as patologias, a candidíase apresentou maior prevalência (29,69\%), seguida pela gengivite $(16,70 \%)$ e queilite angular (14,15\%). Conclusões: Concluímos que 0 exame oral e o diagnóstico precoce da candidíase em pacientes infectados pelo HIV são fundamentais para o tratamento imediato, melhorando a sua qualidade de vida, uma vez que a candidíase é uma lesão bucal muito freqüente nesta população. ntroduction: Strongly associated with Human Immunodeficiency Virus(HIV), oral candidiasis is one of the most common opportunistic infections. Various epedemiological data now emphasize the prevalence of candidiasis in HIV-infected patients and its importance as useful marker for disease progression and prediction for increasing immunossupression. Aim: The purposes of this study were to assess a group of HIV positive patients treated in Heliopólis Hospital, Hosphel - São Paulo, Brazil and refer the oral changing related to the syndrom and compared the results to the literature. Sudy design: Retrospective clinical no randomized. Casuistic and method: Four hundred thirty one HIV+/AIDS brazilian patients (298 men and 133 women) were examined in the Heliópolis Hospital, Hosphel - São Paulo, Brazil in the period from 1995 to 2001 . Results: The most common mean age were 31 to 40 years $(47,10 \%)$, in more than $70 \%$ of the examinated subjects were contaminated through sexual means. Candideasis were the usual infection $(29,69 \%)$, followed by gingivitis $(16,70 \%)$ and angular cheilitis $(14,15 \%)$. Conclusions: O ral lesions occur commonly in HIV infection. A comprehensive oral examination may not only suggest HIV disease but may also be useful in monitoring the disease progression.

\footnotetext{
${ }^{1}$ Cirurgiã-dentista, especialista em periodontia, mestranda pelo Curso de Pós-Graduação em Cirurgia de Cabeça e Pescoço do Hospital Heliópolis, Hosphel, São Paulo.

${ }^{2}$ Cirurgião do Departamento de Cirurgia de Cabeça e Pescoço e $O$ torrinolaringologia do Hospital Heliópolis, Hosphel, São Paulo, Doutor em Medicina pela UNIFESP. ${ }^{3}$ Cirurgiã dentista, chefe do Serviço de Cirurgia Buco-Maxilo-Facial do Hospital Heliópolis - São Paulo, docente em Cirurgia Buco-Maxilo-Facial pela FOUSP. ${ }^{4}$ Cirurgião de Cabeça e Pescoço do Departamento de Cirurgia de Cabeça e Pescoço e O torrinolaringologia do Hospital Heliópolis. Hosphel e Docente Livre da Faculdade de Medicina da Universidade de São Paulo.

Trabalho realizado no Hospital Heliópolis, São Paulo, SP.

Endereço para correspondência: Valdinês Gonçalves dos Santos Cavassani - Rua José Patrício, 43

Rudge Ramos - São Bernardo do Campo - SP - 09601-010

Tel/Fax (0xx11) 4368-9666 - E-mail:hmbpsa comercial@ig.com.br

Artigo recebido em 25 de abril de 2002. Artigo aceito em 8 de agosto de 2002.
} 


\section{INTRODUÇÃO}

A Síndrome da Imunodeficiência Adquirida (AIDS) é caracterizada por severa imunossupressão do hospedeiro, causada pelo Vírus da Imunodeficiência Humana (HIV), manifestando-se por uma grande variedade de sinto mas e sinais clínicos, muitos deles tendo a boca como sítio dessas manifestações.

O espectro das alterações bucais em pacientes HIV positivos é vasto, compreendendo mais de 40 lesões, as quais inúmeras vezes aparecem como as primeiras manifestações da doençâ, ${ }^{1,2}$.

Verifica-se na literatura que há predominância de alguns tipos de lesões, onde a candidíase entre as diversas apresentações clínicas. Depois temos as doenças periodontais, a leucoplasia pilosa, o sarcoma de Kaposi e 0 herpes simples que se situam entre as mais freqüentemente citadas pelos autores ${ }^{3-9}$.

A candidíase é uma infecção fúngica devido à presença de levedura do gênero Candida, o qual é um membro da família Cryptococcaceae; 81 espécies são admitidas no gênero Candida sendo a Candida albicans a mais conhecida e a mais comum ${ }^{10}$.

As infecções fúngicas se instalam em grande número de pacientes portadores do HIV, devido às profundas alterações que ocorrem na função imunológica mediada por linfócitos $T$, com redução da imunidade do paciente ${ }^{11}$.

Q uanto à freqüência da candidíase oral nos pacientes HIV positivos, há variação entre diferentes relatos, mas pode atingir até $94 \%$ dos indivíduos infectados, dependendo do estágio da infecção e da população analisada. Ressaltam a importância da candidíase oral como marcador da progressão da doença e preditivo para o aumento da imunossupressão ${ }^{12}$.

A candidíase oral está associada com a xerostomia, severidade da doença, imunossupressão e idade do paciente acima de 35 anos $^{13}$. É caracterizada por quatro subtipos clínicos como: eritematosa; pseudomembranosa; hiperplásica e queilite angular ${ }^{2}$. A forma eritematosa é representada por áreas avermelhadas, localizadas principalmente no palato, língua e mucosa jugal enquanto a queilite angular acomete as comissuras labiais com variados aspectos clínicos, desde os fissurais a ulcerados, associados ao tipo eritematoso ou pseudo-membranoso. A candidíase hiperplásica representada sob a forma de placas ou nódulos esbranquiçados, firmemente aderidas às áreas eritematosas são menos freqüentes e importantes, podendo ocorrer mais na língua e ser confundida com a leucoplasia pilosa ${ }^{11}$.

Em virtude da alta incidência desta afecção nos portadores de HIV, resolvemos fazer este estudo retrospectivo epidemiológico na casuística do Hospital Heliópolis e compararmos nossos resultados com os da literatura.

\section{CASUÍSTICA E MÉTODO}

A casuística deste estudo epidemiológico retrospectivo constitui-se de 431 prontuários de pacientes portadores do HIV atendidos no Serviço de Estomatologia do Hospital Heliópolis - São Paulo, no período compreendido entre Abril de 1995 a Setembro de 2001.

Os dados são referentes a sexo, raça, faixa etária, formas de contágio e manifestações bucais.

A candidíase bucal, principalmente através das formas pseudomembranosa, eritematosa e a queilite angular, é uma lesão de fácil diagnóstico clínico. Mesmo assim, quando do diagnóstico, foi realizada a citologia esfoliativa ${ }^{10}$. A realização de biópsias não é recomendada por ser o procedimento cirúrgico traumático e desnecessário ${ }^{14}$.

A pesquisa realizada teve aprovação do Comitê de Ética em Pesquisa do Hospital Heliópolis - São Paulo.

Os dados coletados foram reunidos em um programa de computador desenvolvido pelo CDC (Center for Disease Control \& Prevention), o Epilnfo 6.04/2000 que relacionou e analisou estatisticamente através de porcentagem e análise de significância (teste $\mathrm{X}^{2}$ ).

\section{RESULTADOS}

No levantamento dos prontuários dos 431 pacientes portadores do HIV no período de Abril de 1995 a Setembro de 2001, obtivemos dados quanto ao sexo, sendo: 133 mulheres (30,86\%) e 298 homens $(69,14 \%)$ (Gráfico 1 ).

A distribuição dos casos dos pacientes HIV positivos atendidos no Serviço de Estomatologia do Complexo Hospitalar Heliópolis - São Paulo quanto à raça, apresentaram a seguinte distribuição: leucoderma - 307 (71,23\%); melanoderma - 92 (21,35\%); feoderma - $30(6,96 \%)$ e amarelos - 2 casos (0,46\%) (Gráfico 2).

No aspecto da faixa etária, na nossa amostra, variou de 15 a 60 anos, tendo maior incidência na faixa etária compreendida entre 31 a 40 anos, com 203 casos (47,10\%);

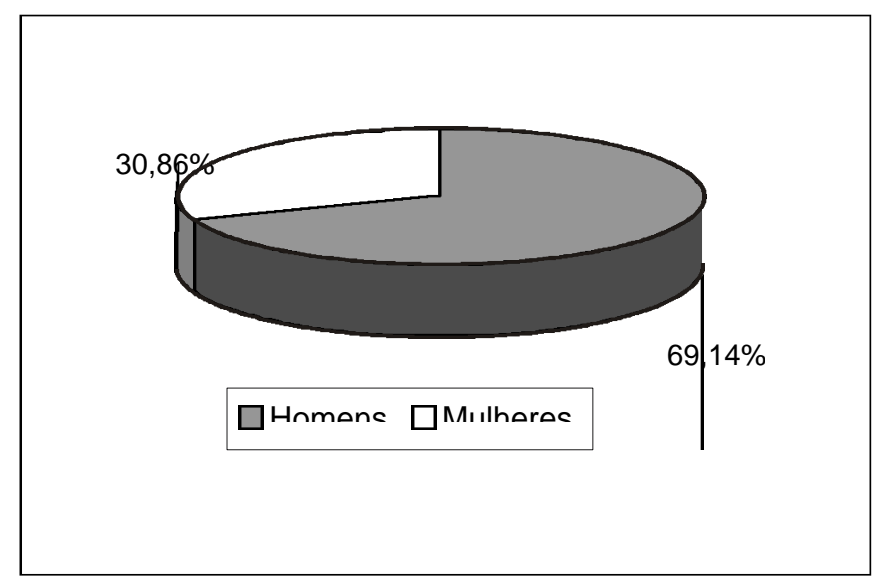

Gráfico 1. Distribuição dos casos por sexo 


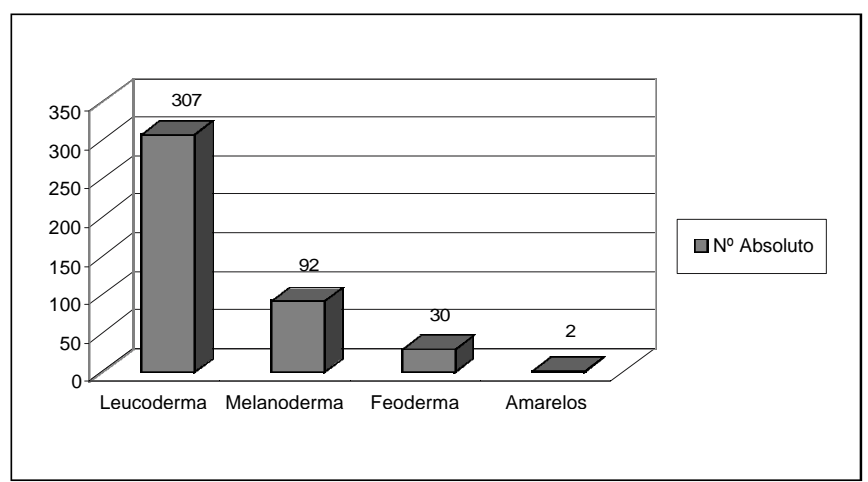

Gráfico 2. Distribuição quanto à raça

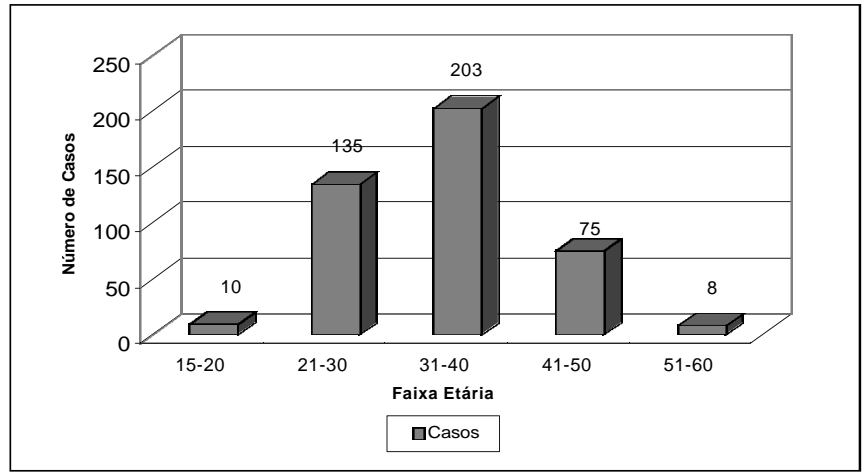

Gráfico 3. Incidência de Casos em função da Faixa Etária

Quadro 1. Manifestações Estomatológicas

\begin{tabular}{|lccc|}
\hline Doenças & $\begin{array}{c}\text { No } \\
\text { Absoluto }\end{array}$ & $\begin{array}{c}\text { № } \\
\text { Percentual }\end{array}$ & $\begin{array}{c}\text { Localização } \\
\text { mais freqüente }\end{array}$ \\
\hline Candidíase oral & 128 & 29,69 & \\
- Pseudomembranosa & 78 & 18,09 & Língua e Palato \\
- Eritematosa & 48 & 11,13 & Palato \\
- Leucoplásica & 2 & 0,46 & Palato \\
Gengivite & 72 & 16,70 & Generalizada \\
Queilite Angular & 61 & 14,15 & Comissura labial \\
Ausentes & 110 & 25,52 & Sem manifestações \\
\hline
\end{tabular}

seguida pela faixa de 21 a 30 anos com 135 casos (31,32\%); 41 a 50 anos com 75 casos (17,40\%); 15 a 20 anos com 10 casos (2,32\%) e 51 a 60 anos com 8 casos (1,85\%) (G ráfico 3). obtivemos:

Em relação às formas de contágio na nossa amostra

- contágio por via sexual - 333 casos (71,26\%);

- usuários de drogas injetáveis - 88 casos $(20,42 \%)$;

- transfusão sanguínea e hemoderivados - 6 casos (1,39\%);

- vertical (criança nascida de mãe infectada) - 1 caso $(0,24 \%) ;$

- via de transmissão não referida - 3 casos (0,63\%) (Gráfico 4).

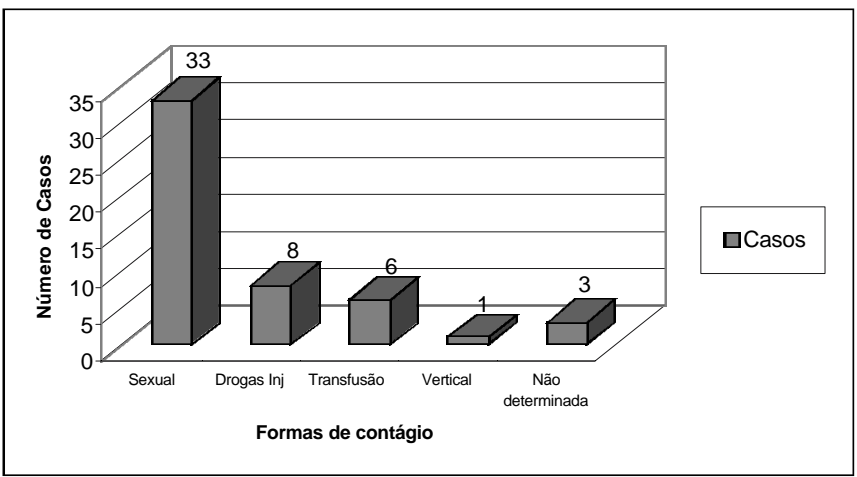

Gráfico 4. Formas de Contágio

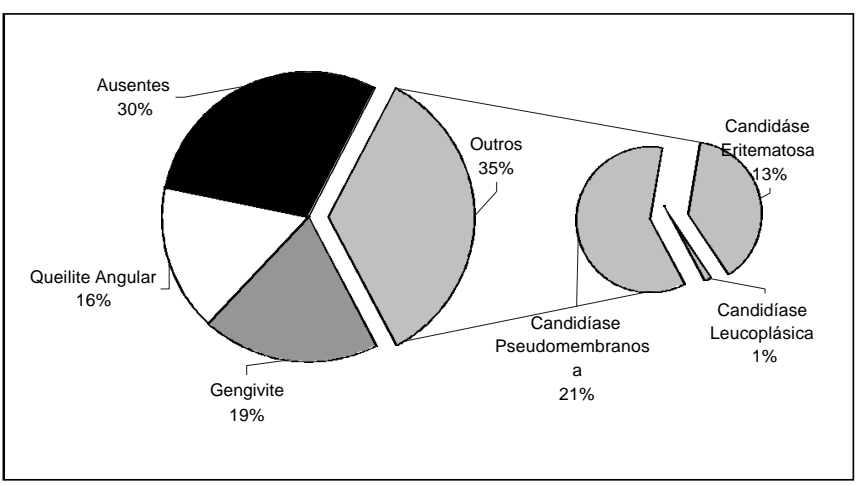

Gráfico 5. Manifestações Estomatológicas

No que se refere às manifestações estomatológicas encontramos: 128 casos de candidíase oral, sendo a manifestação de maior incidência com 78 casos de candidíase pseudomembranosa (com a localização mais freqüente na língua e palato), 48 casos de candidíase eritematosa (com localizações no palato) e 2 casos de candidíase leucoplásica (localizadas no palato). Na nossa amostra, a gengivite se mostra em 72 casos localizada de forma generalizada. A queilite angular com localização na comissura labial surge em terceiro lugar com 61 casos, sendo que 110 pacientes não apresentaram nenhuma manifestação bucal (Q uadro 1 e G ráfico 5).

Todos os dados analisados tiveram valor estatisticamente significante ao nível de $1 \%(p<0,01)$.

\section{DISCUSSÃO}

O Serviço de Estomatologia do Hospital Heliópolis está relacionado diretamente à clínica de moléstias infectocontagiosas do Hospital Heliópolis e como esta não é referência para os casos de pediatria, o número de crianças em nosso levantamento foi apenas seis, não sendo computados no total de 431 casos. 
A distribuição etária é muito característica, sendo nossos resultados na faixa etária dos 20 aos 40 anos a mais freqüente. Eles estão de acordo com outros autores ${ }^{9}$ que, analisando 160 pacientes, constataram que a média de idade foi de 35,5 anos. Como o grupo etário dos 20 aos 40 anos corresponde ao período de maior atividade sexual torna-se compreensível o porquê da maior parte dos infectados pertencerem a esta faixa de idade.

Q uanto à forma de contágio, a principal ainda foi a homossexual em 12,99\% (56 casos), concordante com a literatura ${ }^{15-18}$. Porém, é relevante salientar que a via heterossexual cresceu muito nos últimos anos, sendo potencialmente importante em homens e mulheres. Em seguida encontramos os viciados em drogas injetáveis ${ }^{19}$, fato aferido em $20,42 \%$ de nossa casuística.

Nos 431 pacientes avaliados, as manifestações bucais relatadas são as verificadas na primeira consulta no Serviço de Estomatologia. Encontramos 110 casos assinto máticose sem lesão bucal no exame clínico $(25,52 \%)$. Os restantes $221(74,48 \%)$ apresentavam lesão bucal. Estes fatos já foram relatados na literatura e com incidências semelhantes às nossas $9,11,16,20$.

A incidência das formas da candidíase esteve de acordo com a literatura, onde, em ordem decrescente, encontram-se a psedomembranosa, queilite angular e eritematosa ${ }^{89,202023}$. De acordo com a nossa casuística, a queilite angular revelou uma elevada incidência - 61 casos (14,15\%), quando comparada aos achados de literatura, que foram muito inferiores $s^{9,10,21,23}$.

Em nosso estudo foram observadas associações de formas de candidíase e presença em múltiplos sítios bucais. A causa pode estar relacionadaà imunossupressão causada pela diminuiç̧ão dos linfócitos CD 4 e a fatores locais, dentre os quais, a xerostomia.

A xerostomia é a diminuição da secreção salivar e é freqüentemente contemplada em indivíduos HIV positivos, podendo favorecer traumas teciduais, condição importante para a infecção com Candida e diminuição de proteínas antimicrobiais ${ }^{13,24-26}$. Todavia, após a introdução de terapêuticas medicamentosas anti-retrovirais houve diminuição das manifestações bucais. Em virtude de nossos casos já estarem sendo tratados quando nos chegam, talvez seja esta a explicação do grande número de ausência de lesão em 110 casos (25,52\%).

A candidíase bucal, principalmente através das formas pseudomembranosa eritematosa e a queilite angular, é uma lesão de fácil diagnóstico clínico. Mesmo assim, quando necessários exames laboratoriais para configuração do diagnóstico, foi realizada a citologia esfoliativa ${ }^{10}$. A realização de biópsias não é recomendada por ser o procedimento cirúrgico traumático e desnecessário ${ }^{14}$.

0 tratamento odontológico dos pacientes soropositivos para HIV deve ser sempre planejado e com acompanhamento médico. Com o início da terapia anti-retroviral altamente potente (HAART) alguns pesquisadores verifica- ram a redução acentuada na ocorrência de infecções oportunistas. A prevalência das manifestações bucais também sofreu decréscimo significativo com o advento da HAART ${ }^{26,27}$. A incidência de candidíase, leucoplasia pilosa e doenças periodontais diminuíram; em oposição, os pacientes apresentaram aumento nas doenças das glândulas salivares ${ }^{26,27}$.

É importante o conhecimento das manifestações bucais em pacientes HIV positivos pelo profissional da saúde para o diagnóstico, tratamento e conseqüente melhora da qualidade de vida destes pacientes.

\section{CONCLUSÕES}

Em nossa casuística, houve prevalência de candidíase pseudomembranosa como lesão associada à Síndrome de Imunodeficiência Adquirida, em faixa etária predominante dos 31 aos 40 anos. A forma de contágio predominante foi por via sexual e ainda em nossa amostra houve maior presença de homens leucodermas.

\section{REFERÊNCIASBIBUOGRÁFICAS}

1. Moniaci D, Greco D, Flecchia $G$ et al. Epidemiology,clinical features and prognostic value of HIV- 1 related oral lesions. J Oral Pathol Med 1990;19:477-81.

2. Nazanin N, Epstein JB. Classification of oral lesions in HIV infection. J Clin Periodontol 2001;28:137-45.

3. Arendorf TM, Bredekamp B, Coete CCA et al. Oral manifestations of HIV infection in 600 South African patients. J Oral Pathol Med 1998;27:176-9.

4. Axell T, Baert S, Brocherion C et al. Revised classification of HIVassociated oral lesion. Britden J 1991;170:305-6.

5. Barone R, Ficarra G, Gagliotti D et al. Prevalence of oral lesions among HIV infected intravenous drug abuses and other risk groups. Oral Surg 1990;69:169-73.

6. Ceballos-Salobrena A, Aguirre-Urizar JM, Bagan-Sebastian JV. Oral manifestations associated with human imunodeficiency virus infection in a Spanish population. J Oral Pathol Med 1996;25:523-6.

7. Epstein J, Scully C. HIV infection: clinical features and treatment of thirty-three homossexual men with Kaposis sarcoma. Oral Surg 1991;71:38-41.

8. Greenspan D, Greenspan JS. Managment of the Oral Lesions of HIV Infection. J Am Dent Assoc 1991;122:26.

9. Laskaris G, Hadjivassiliov M, Stratigos J. Oral signs and symptoms in 160 HIV-infected patients. J Oral Path Med 1992;21:120-3.

10. Samaranayake LP. Oral mycoses in HIV infection. Oral Surg Oral Med Oral Pathol 1992;73:113.

11. Lima OCC, Silveira FRX, Birman EG. Manifestações bucais de origem infecciosa em pacientes HIV-positivos ou com AIDS/ Doenças fúngicas. Rev ABO Nac 1994;2:28-32.

12. Mesquita RA, Aguiar MCF, Tarquino SBC et al. Candidíase oral ca infecção HIV. Rev CROMG 1998:4:27-31.

13. Mc Carthy GM. Host factors associated with HIV-related oral candidiasis. Oral Surg Oral Med 1992;73:181-6.

14. Glick M. Evaluation of prognosis and survival of the HIV-infected patient. Oral Surg Oral Med Oral Pathol 1992;74:386-92.

15. Epstein JB, Silverman SJ, Gorsky M. The continuing epidemic of HIV and AIDS. J Am Dent Assoc 1998:64:426-30.

16. Lozada F, Silverman Jr, Migliorati CA et al. O ral manifestations of tumor and opportunitic infections in the acquired immunodeficiencyu syndrome (AIDS): Findings in 53 homossexual men with Kaposi's sarcoma. Oral SurgOral Med Oral Pathol 1983;56:491-4. 
17. Phelan JA, Begg MD, Lamster IB. O ral candidiasis in HIV infection: predictive value and comparison of findings in infecting drug uses and homossexual men. J Oral Pathol Med 1997;26:237-43.

18. Souza LB, Pinto LP, Medeiros AMC. Manifestações orais em pacientes com AIDS em uma população brasileira. Pesq Odont Bras 2000;4:79-85.

19. Boletim Epidemiológico De Aids Do Ministério Da Saúde, $\mathrm{n}^{\circ}$ $19,2000$.

20. Van Der Wall I, Schulten GAJM, Pindborg JJ. Oral manifestations of AIDS: an overview. Int Dent J 1991;41:3-8.

21. Jaeger MMM, Araújo NS. Candidíase bucal em pacientes infectados pelo HIV. Rev Fac Odont FZL 1990;2:89-97.

22. Nielsen $H$, Bentsen $K D, H o j t v e d ~ C$ et al. Oral candidiasis and immune status of HIV-infected patients. J Oral Pathol Med 1994;23:140-3.
23. Panganathan K, Reddy BVR, Kumarasamy $\mathrm{N}$ et al. Oral lesions and conditions associated with human immunodeficiency virus infection in 300 south Indian patients. O ral Dis 2000;6:152-7.

24. Bentsen NH, Hojtved L, Willewoes EH et al. Oral candidiasis and immune status of HIV: infected patients. J Oral Pathol Med 1994;23:140-3.

25. Hodgson TA. HIV-associated oral lesions; Prevalence in Zambia. Oral Dis 1997;3:546-50.

26. Patton LL, Mc Kaig RG, Strauss RP et al. Oral manifestations of HIV in a southeast USA population. Oral Dis 1998;4:164-9.

27. Arribas JR, Hernandez-Albujar S, Gonzales-Garcia JJ, Pena JM, Gonzales A, Canedo T, Madero R,Vazquez JJ, Pow derly WG. Impact of protease inhibitor therapy on HIV-related oropharyngeal candidiasis. AIDS 2000;14(8):979-85.

28. Greenwood I, Heylen R, Zakrzewska JM. Anti-retroviral drugs implications for dental prcribing. Brit Dent J 1998;184:478-82. 